\title{
Filter Features Extraction from Optical Spectra
}

\author{
Fabiano Locatelli( ${ }^{(1)}, K_{\text {Konstantinos Christodoulopoulos }}^{(2)}$, Josep M. Fàbrega( ${ }^{(1)}$, Michela Svaluto

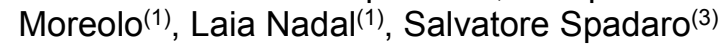 \\ (1) Centre Tecnològic de Telecomunicacions de Catalunya (CTTC/CERCA), Castelldefels (Barcelona), \\ Spain, fabiano.locatelli@cttc.es \\ (2) Nokia Bell Labs, Stuttgart, Germany \\ (3) Universitat Politècnica de Catalunya (UPC), Barcelona, Spain
}

\begin{abstract}
We propose a method to estimate the central frequency and the bandwidth of optical filters, that relies on optical spectra captured with optical channel monitors at ROADM's ingress port. We demonstrate for both features high estimation accuracy using simulated and experimental data.
\end{abstract}

\section{Introduction}

Ideally, in an optical network, we would like to have omnipresent and powerful optical performance monitors (OPMs ${ }^{[1]}$. In this way, various parameters can be monitored across the network, enabling advanced failure identification and localization operations ${ }^{[2]}$. This also allows to improve the network efficiency through e.g. a better estimation of the quality of transmission (QOT) and to adjust the margins of existing and future connections ${ }^{[3]}$. Specifically, the optical signal-to-noise ratio (OSNR) and the filter related features, such as the filter shape, $3 \mathrm{~dB} / 6 \mathrm{~dB}$ bandwidth (BW) and shift with respect to the central frequency, are among the most important parameters to be estimated with $\mathrm{OPM}^{[4]}$. Moreover, a disaggregated network requires advanced monitoring capabilities to cope with the expected specification mismatches and performance variations of the network elements provided by different vendors ${ }^{[5]}$.

In reality, having omnipresent and powerful OPMs is not a feasible solution because of its high cost. To reduce it, cheap monitors must be chosen also limiting their placement. Hence, we propose the use of cheap optical spectrum analyzers (OSAs) as OPMs, such as the optical channel monitors $(\mathrm{OCMs})^{[6]}$. OCMs are solutions which combine low cost (order of $1 \mathrm{~K}$ euros) with good spectral resolution $(1 \mathrm{GHz}$ down to 600 $\mathrm{MHz}$ ). The most appropriate position for the OCMs is close to the reconfigurable optical add/drop multiplexers (ROADMs), which are key enablers in today's core and metro networks ${ }^{[7]}$. The ROADMs include optical filters, which often completely remove any trace of the noise at the signal sides, making the OSNR estimation very challenging ${ }^{[8]}$. In addition, due to the ageing of the network, the presence of multivendor elements (i.e., disaggregated networks) or simply because of a failure, the optical filters can introduce optical impairments ${ }^{[9]}$, such as the filter/laser misalignment and the filter cascade effect (FCE).

We identified 3 scenarios for the placement of the OCMs with respect to the ROADMs, as depicted in Fig. 1. The first scenario, considers the monitors to be located at the ingress ports of each ROADMs, i.e. before their ingress wavelength selective switches (WSSs); while the second scenario, at the ROADMs egress ports. A third scenario, which will be referred to as sparse, considers a non-uniform OCMs placement, that is, some nodes are equipped with monitoring only at their input, some others only at their output, some at both and some not monitored at all. These three scenarios also apply in the disaggregated network case: choosing one of them would allow lower costs, while providing the required monitoring functionalities for the network to operate properly. In both aggregated and disaggregated cases, according to the chosen scenario, the pool of monitorable features varies. In the first one, placement at the ingress, all the features of the signal entering the node, such as its central frequency, 3/6-dB BW and the amplified spontaneous emission (ASE) noise level, are easily retrievable. On the other hand, if we consider the second scenario, placement at the egress, the detection of the aforementioned signal information is made difficult by the filtering. Nevertheless, filter features such as filter central frequency (i.e. shift of the filter) and 3/6-dB BW, can be easily detected.

In our previous work ${ }^{[10]}$, we proposed a machine learning (ML)-based method, which exploited optical spectra for estimating the ASE noise. In that paper, we studied the egress OCMs scenario (scenario 2 in Fig. 1), while in this work we focus on the ingress scenario (scenario 1). Exploiting the spectra captured by such OCMs, we propose a method to retrieve the optical filter's features, including its central frequency and its 3/6-dB BW. We test the accuracy of the proposed solution with spectral data acquired from simulations and experiments.

\section{Network architecture and spectral model}

The proposed network architecture is depicted in Fig. 1. In particular, we denote by $n$ a generic 


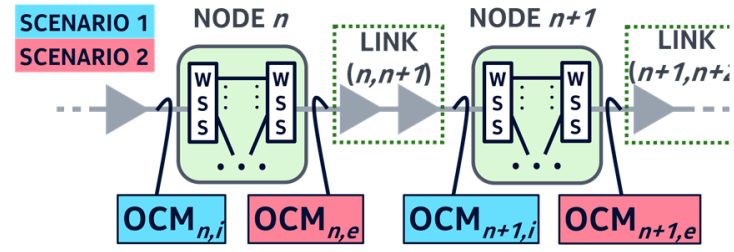

Fig. 1: Proposed network architecture. WSS: wavelength selective switch; OCM: Optical channel monitor.

node of the optical network and by $n+1$ one of its adjacent nodes. We also denote by $\mathrm{OCM}_{n, i}$, $\mathrm{OCM}_{n+1, i}, \quad \mathrm{OCM}_{n, e}$ and $\mathrm{OCM}_{n+1, e}$ the optical channel monitors placed at the ingress and at the egress of these nodes, respectively. Finally, we denote the link, consisting of a single or multiple optical fiber spans and optical amplifiers connecting the two nodes, as $(n, n+1)$. Typical ROADM architectures employ 1 or 2 filters to switch bypassing connections. We treat both cases as the same: if 2 filters are involved, we model an equivalent for the specific ingressegress direction. To estimate any features of a ROADM filter, as a first step, the spectrum of its transfer function (TF) must be retrieved. To do so, in an ideal scenario (where the nodes are equipped with omnipresent OCMs), for node $n$ we would divide, in the linear domain, the power spectral density captured by $\mathrm{OCM}_{n, e}$ and the one captured by $\mathrm{OCM}_{n, i .}$. Since the scenario we are considering does not include an OPM placed after node $n$, we use the spectra captured by $\mathrm{OCM}_{n+1, i}$. The result of this division, represents the spectrum of the filter TF plus the noise accumulated over the link $(n, n+1)$. Therefore, to reduce this unwanted contribution, we can estimate the ASE noise and remove it from $\mathrm{OCM}_{n+1, i}$ spectra. In fact, the filter at node $n$ suppresses the noise introduced by the links before it. Thus, the noise at the signal's sides of the spectra collected after the link $(n, n+1)$, is mainly due to the contribution of that same link.

The authors $\mathrm{in}^{[11]}$, proposed a model to characterize the optical field spectrum $S(f)$ of a bandpass filter created by a WSS, using the error function $\operatorname{erf}(x)$. We extended this model to consider possible nonzero power reference and shifts of the filter. The optical field spectrum that we modelled is as follows:

$$
\begin{aligned}
S(f)=\frac{1}{2} A \sqrt{2 \pi} & {\left[\operatorname{erf}\left(\frac{B / 2-f-D}{\sqrt{2} A}\right)\right.} \\
& \left.-\operatorname{erf}\left(\frac{-B / 2-f-D}{\sqrt{2} A}\right)\right]+C
\end{aligned}
$$

where $f$ represents the frequency, $A$ is the parameter related to the steepness of the filter edges (i.e. the filter order), $B$ represents the $6-\mathrm{dB}$ BW of the filter, $C$ is a correction factor for

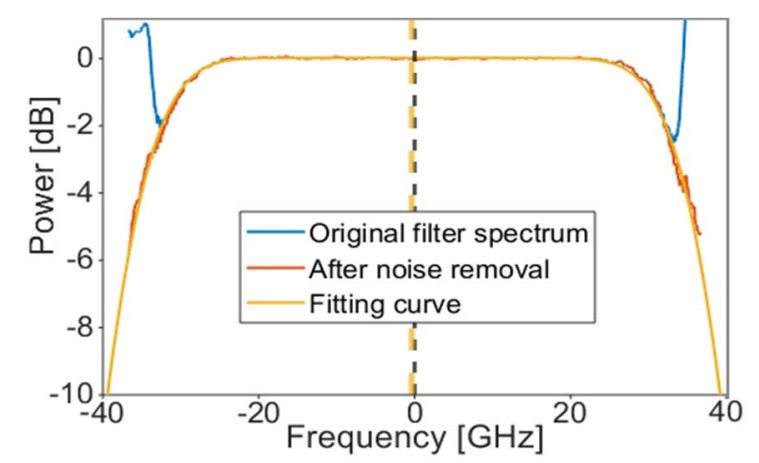

Fig. 2: Original (blue) and after noise removal (orange) filter spectra with the fitting curve (yellow). The dotted yellow line represents the shifted central frequency with respect to the reference (black dotted line).

eventual $y$-axis shifts and $D$ is the parameter that corrects the filter central frequency shift. As shown in Fig. 2, the filter TF spectrum found by the division may not reach deep values since at the spectra edges we processed noise and not channel's signal. Therefore, to enable the $6-\mathrm{dB}$ filter BW evaluation, we first have to estimate its full TF. Hence, we can tune $A, B, C$ and $D$ such that the squared and logarithmic version of Eq. (1) fits the available portion of filter TF spectrum, as depicted in Fig. 2. For a better estimation of the fitting curve, we define a relatively small range for the four parameters (e.g. from the filter specifications). Once we calculate the fitting curve, we can easily estimate the 6-dB filter BW by checking the values assigned to $B$. On the other hand, the parameter $D$ provides an estimation of the filter central frequency shift. We also identified other options to estimate the filter features, such as the centre of mass (for frequency shift) or ML algorithms like Gaussian process regression (GPR), but none had better accuracy than the model based on Eq. (1).

\section{Simulation, experimental setup and results}

For simulations, we used the VPIphotonics setup shown in Fig. 3a. We generated a $32 \mathrm{GBd}$ polarization multiplexed-quadrature phase shift keying (PM-QPSK) modulated signal, with 0.1 roll-off factor. After the transmitter (TX), we simulate an optical link cascading a number of 80 $\mathrm{km}$ length fiber spans and erbium-doped fiber amplifiers (EDFAs). Each link is followed by an optical node, which we implemented as a cascade of 2 optical filters, with $2^{\text {nd }}$ order Gaussian TF. We assumed three different 6-dB filter BW values: $36.5,37.5$ and $38.5 \mathrm{GHz}$. In addition, to emulate the laser drift/filter shift, we shifted the central frequency of each filter with values ranging between -1 and $+2 \mathrm{GHz}$. For simplicity, we assumed that the optical filters of each node had the same characteristics. Finally, at the input of every node, we placed an OCM ( $1 \mathrm{GHz}$ resolution) through which we collected the 


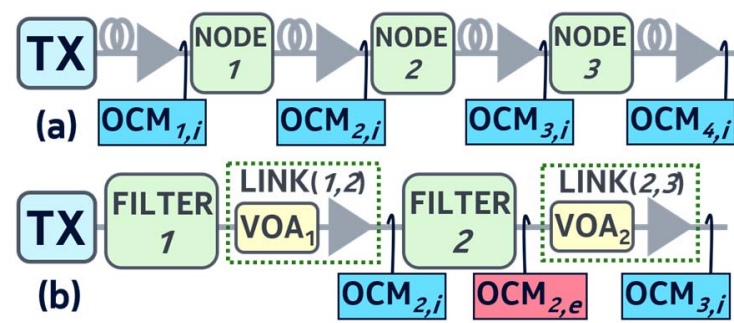

Fig. 3: (a) Simulation setup. TX: transmitter; OCM: optical channel monitor. (b) Experimental setup. VOA: variable optical attenuator.

set of spectra to test our algorithm.

To further validate the proposed approach, we also collected a set of optical spectra through the experimental setup depicted in Fig. 3b. We generated a 64 GBd PM-QPSK modulated signal, with 0.1 roll-off factor. After the TX, in order to emulate the FCE over the previous part of the path, we placed an optical filter and we varied its $\mathrm{BW}$ and central frequency with respect to the TX. Following the first filter, in order to emulate different fiber span lengths, we cascaded a variable optical attenuator (VOA) and an EDFA, which represented link $(1,2)$. After that link, we placed another optical filter, namely Filter 2 , that corresponded to the filter on which we tested our model. We set Filter 2 TF as rectangular and varied its $6-\mathrm{dB}$ BW and its central frequency. At the ingress and at the egress port of Filter 2, we placed two OCMs: $\mathrm{OCM}_{2, i}$ and $\mathrm{OCM}_{2, e}$, respectively. Although we focused on scenario 1 (i.e. the ingress scenario), we placed $\mathrm{OCM}_{2, e}$ to retrieve Filter 2 ideal TF, which we used as reference for our tests. Furthermore, after Filter 2, we cascaded a second link, namely link $(2,3)$, consisting of $\mathrm{VOA}_{2}$ and an EDFA and varied $\mathrm{VOA}_{2}$ 's attenuation. Finally, we placed $\mathrm{OCM}_{3, i}$, after link (2,3). We formed 3 cases (sets) by varying $\mathrm{VOA}_{1}, \mathrm{VOA}_{2}$ and Filter 1 values, while keeping Filter 2 values fixed. The considered combinations are summarized in Tab. 1. For all simulation and experimental scenarios, we set the OCMs resolution at $1 \mathrm{GHz}$.

We first retrieved the TFs of Node 1, Node 2 and Node 3 in the simulation setup and of Filter 2 in the experimental setup, with the procedure explained in the previous section and then we applied the algorithm for the filter central frequency and 6-dB BW estimation. In Tab. 2 we list the standard deviation $(\sigma)$, the mean squared error (MSE) and the maximum absolute error (MAE) for the two considered filters features, for both simulations and experiments.

As the signal crosses more links and filters, its shape is distorted and more affected by the noise. In the simulations, we observed that the third filter TF had a narrower and noisier shape compared to the first one. In fact, our process
Tab. 1: Considered cases for the experimental setup

\begin{tabular}{|c|c|c|c|c|}
\hline Case & $\begin{array}{c}\text { Filter 1 } \\
\text { 6-dB BW } \\
\text { (shift) } \\
\text { [GHz] }\end{array}$ & $\begin{array}{c}\text { Link(1,2) } \\
\text { VOA }_{1} \\
\text { [dB] }\end{array}$ & $\begin{array}{c}\text { Filter 2 } \\
\text { 6-dB BW } \\
\text { (shift) } \\
\text { [GHz] }\end{array}$ & $\begin{array}{c}\text { Link(2,3) } \\
\text { VOA } \\
\text { [dB] }\end{array}$ \\
\hline 1 & $74(-2)$ & 0 & $\begin{array}{c}73,75,77 \\
(-1,0,+1)\end{array}$ & 10 \\
\hline 2 & $69(-2)$ & 10 & $\begin{array}{c}73,75,77 \\
(-1,0,+1)\end{array}$ & 10 \\
\hline 3 & $74(-2)$ & 0 & $\begin{array}{c}73,75,77 \\
(-1,0,+1)\end{array}$ & 20 \\
\hline
\end{tabular}

Tab. 2: Filters 6-dB BW and shift estimation errors

\begin{tabular}{|c|c|c|c|c|}
\hline & Filter & Metric & $\begin{array}{c}\text { 6-dB } \\
\text { BW est. }\end{array}$ & $\begin{array}{l}\text { Shift } \\
\text { est. }\end{array}$ \\
\hline \multirow{9}{*}{ 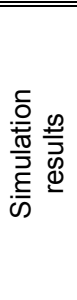 } & \multirow{3}{*}{ Node 1} & $\bar{\sigma} \sigma[\mathrm{GHz}]$ & 0.0807 & 0.0334 \\
\hline & & MSE & 0.0183 & 0.0019 \\
\hline & & MAE [GHz] & 0.1937 & 0.0655 \\
\hline & \multirow{3}{*}{ Node 2} & $\sigma[\mathrm{GHz}]$ & 0.0479 & 0.0178 \\
\hline & & MSE & 0.0024 & 0.0008 \\
\hline & & MAE [GHz] & 0.1057 & 0.0454 \\
\hline & \multirow{3}{*}{ Node 3} & $\sigma[\mathrm{GHz}]$ & 0.1247 & 0.0482 \\
\hline & & MSE & 0.0163 & 0.0026 \\
\hline & & MAE [GHz] & 0.2962 & 0.0997 \\
\hline \multirow{9}{*}{ 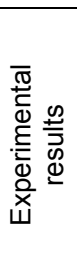 } & \multirow{3}{*}{$\begin{array}{c}\text { Filter } 2 \\
\text { (Case 1) }\end{array}$} & $\sigma[\mathrm{GHz}]$ & 0.1433 & 0.1341 \\
\hline & & MSE & 0.0204 & 0.0228 \\
\hline & & MAE [GHz] & 0.3933 & 0.2433 \\
\hline & \multirow{3}{*}{$\begin{array}{c}\text { Filter } 2 \\
\text { (Case 2) }\end{array}$} & $\sigma[\mathrm{GHz}]$ & 0.4508 & 0.2256 \\
\hline & & MSE & 0.1978 & 0.0597 \\
\hline & & MAE [GHz] & 0.6811 & 0.5054 \\
\hline & \multirow{3}{*}{$\begin{array}{c}\text { Filter } 2 \\
\text { (Case 3) }\end{array}$} & $\sigma[\mathrm{GHz}]$ & 0.3470 & 0.1018 \\
\hline & & MSE & 0.1383 & 0.0133 \\
\hline & & MAE [GHz] & 0.7627 & 0.1863 \\
\hline
\end{tabular}

uses the previous OCM spectra as reference to find the next filter features, nullifying the effect of the path up to that point. However, there is some residual error which increases as links/filters are added. Therefore, the filter features estimation accuracy slightly deteriorates as the number of links increases (still showing excellent approximations up to 3 nodes). Likewise, experimental results showed lower accuracy for narrower Filter $1 \mathrm{BW}$ and higher $\mathrm{VOA}_{1}$ attenuation (case 2, which emulates bigger FCE) and for higher $\mathrm{VOA}_{2}$ attenuation (case 3, which emulates longer spans). Note that we did not observe improvements in the results using a better OCM resolution (600 MHz).

\section{Conclusions}

We presented a method for estimating optical filter features in a network where OCMs are present at the input of the optical nodes. We observed excellent accuracy for the central frequency (<0.03 MSE) and the 6-dB BW $(<0.02$ MSE) estimation with simulated spectra and very good accuracy (<0.06 and $<0.2 \mathrm{MSE}$ ) with experimental spectra. Future plans include the study of the sparse monitoring scenario.

\section{Acknowledgements}

This work was funded by the ONFIRE project supported by the EU Horizon 2020 research and innovation programme under the Marie Skłodowska-Curie grant agreement No. 765275. The authors would like to thank Fred Buchali, Theresa Hu, Karsten Schuh and Ankush Mahajan for lab support and fruitful discussions. 


\section{References}

[1] Z. Dong et al., "Optical Performance Monitoring: A Review of Current and Future Technologies," in IEEE/OSA Journal of Lightwave Technology, vol. 34, no. 2, pp. 525-543, 2016.

[2] B. Shariati, M. Ruiz, J. Comellas, and L. Velasco, "Learning From the Optical Spectrum: Failure and Detection and Identification," in IEEE/OSA Journal of Lightwave Technology, vol. 37, no. 2, pp. 433-440, 2019.

[3] K. Christodoulopoulos et al., "Toward efficient, reliable, and autonomous optical networks: the ORCHESTRA solution [Invited]," in IEEE/OSA Journal of Optical Communications and Networking, vol. 11, no. 9, pp. C10-C24, Sep. 2019.

[4] C. Delezoide, P. Ramantanis, and P. Layec, "On the Performance Prediction of Optical Transmission Systems in Presence of Filtering," in 19th International Conference on Transparent Optical Networks (ICTON), 2017.

[5] M. Filer et al., "Multi-Vendor Experimental Validation of an Open Source QoT Estimator for Optical Networks," in IEEE/OSA Journal of Lightwave Technology, vol. 36, no. 15, pp. 3073-3082, Aug. 2018.

[6] Finisar, "Flexgrid High Resolution Optical Channel Monitor (OCM)," 2015. [Online]. Available: https://optical.communications.ii-vi.com/roadmswavelength-management/focm $01 \mathrm{fxc} 1 \mathrm{mn}$

[7] B. Collings, "New Devices Enabling Software-Defined Optical Networks," IEEE Communication Magazine, vol. 51, no. 3, pp. 66-71, Mar. 2013.

[8] A. Morea, J. Renaudier, T. Zami, A. Ghazisaeidi, and O. Bertran-Pardo, "Throughput Comparison Between $50-\mathrm{GHz}$ and $37.5-\mathrm{GHz}$ Grid Transparent Networks [Invited]," IEEE/OSA Journal of Optical Communications and Networking, vol. 7, no. 2, pp. A293-A300, Feb. 2015.

[9] J. M. Fàbrega et al., "On the filter narrowing issues in elastic optical networks," IEEE/OSA Journal of Optical Communications and Networking, vol. 8, no. 7, pp. A23-A33, Jul. 2016.

[10] F. Locatelli et al. "Experimental Demonstration of a Machine Learning-Based in-band OSNR Estimator from Optical Spectra," in Optical Network Design and Modelling (ONDM) International Conference, 2020.

[11] C. Pulikkaseril et al., "Spectral modeling of channel band shapes in wavelength selective switches," in Optic Express, vol. 19, no. 9, pp. 8458-8470, 2011. 